\title{
Perception of two-component electrocutaneous stimuli
}

\author{
HALLIDAY J. H. MacFIE and DAVID M. H. THOMSON \\ Agricultural Research Council, Meat Research Institute \\ Langford Nr. Bristol, Avon BS18 7DY, England
}

\begin{abstract}
Electrocutaneous stimuli varying in strength $(6,8,10 \mathrm{~V})$ and frequency $(9,27,81 \mathrm{~Hz})$ were presented in pairs. Subjects were asked to give a numerical score to represent their perception of overall difference in a pair. To assess their ability to use numbers and their performance on unfamiliar stimuli, no guidance on the nature of the stimuli or the range or scale of the scores was given. Dimensional analysis indicated that perception of differences in one component was influenced by the absolute level of the other component, if this was constant in the pair, or by the difference. Considerable variation among subjects in the nature and extent of this interference effect was observed. Individual differences scaling (INDSCAL) enabled a satisfactory consensus model to be obtained. An empirical relationship expressing the observed twocomponent difference scores in terms of one-component scores was derived. Increasing voltage level diminished perception of frequency difference. Higher frequency levels augmented perception of voltage difference. This relationship is a modification of the combinatorial model proposed by Krantz and Tversky (1975). Individual differences in the combinatorial process are allowed for by application of the subject weightings from the INDSCAL solution.
\end{abstract}

A working mathematical model of the process by which humans combine a number of simultaneously perceived difference components between two stimuli is a necessary precursor to the routine use of sensory panels to evaluate possibly unfamiliar or nonverbalized stimuli. In this paper, we report the use of electrocutaneous stimuli that vary in both pulsed repetition frequency and voltage to provide a data base for the investigation and model building of the process by which subjects organize and compare multidimensional stimuli.

Recent studies suggest that the perception of such stimuli may be characterized as one of three behavior modes. If the components of a stimulus are completely analyzable (discrete psychological dimensions), subjects are able to attend to each component separately. The perceived difference between any two stimuli will thus approximate the sum of the differences perceived along each dimension. At the opposite extreme, the stimulus components may be completely nonanalyzable (interactive) and differences between stimuli are perceived along a single complex dimension. The third behavioral mode is characterized by the perception of both discrete and interactive stimulus components.

When comparing pairs of multicomponent stimuli from within a given stimulus set, all three behavioral

The authors wish to thank R. W. R. York, Electronics Section, Meat Research Institute, who designed and constructed the electrical apparatus. D. M. H. Thomson (whose present address is Department of Food Science, University of Reading, Reading, Berkshire, England) was the recipient of a Research Studentship from the Agricuitural Research Council, England. modes may be apparent within the judgments made by a single subject who may attend to the various stimulus components differently, depending on the comparison being made.

The analyzability of stimulus components, and hence the behavioral mode of a subject, can be assessed by comparing the "goodness of fit" of multidimensional scaling solutions having different Minkowski metrics (Shepard, 1964). City block metric $(r=1.0)$ should render a superior fit when the dimensions are completely analyzable, whereas nonanalyzable dimensions should be fitted best by a Euclidean metric. Shepard postulated metrics intermediate between city block and Euclidean for stimulus sets having partially analyzable dimensions.

As an alternative, Tversky and Krantz proposed an additive difference model of dimensional organization (termed dimensional analyses henceforth), in which two criteria defining analyzability were imposed (Krantz \& Tversky, 1975; Tversky \& Krantz, 1969,1970 ). The first criterion, termed interdimensional additivity, requires that differences perceived along a particular component dimension should be completely independent of differences in any other component. The second criterion, intradimensional subtractivity, requires that the contribution of any component to the perceived overall difference should be directly related to the actual difference present in that dimension.

If these criteria cannot be upheld, then further, more sophisticated analyses are necessary to investigate the nature and extent of dimensional interaction. For example, using dimensional analysis, 
more complex models may be derived which still retain the components of the stimuli as psychological dimensions but take account of interaction (Krantz \& Tversky, 1975). Multidimensional scaling procedures such as individual differences scaling analysis (INDSCAL, Carroll \& Chang, 1970) may also be used to this effect. INDSCAL retains the additivity and subtractivity assumptions and produces a configuration of the stimuli in multidimensional Euclidean space. Interactions between the components may be revealed by unequal distances among the stimuli or by the presence of important extra dimensions in the scaling solutions, although the work of Cohen and Jones (1974) on subsampling of dimensions suggests that the latter is less likely.

Comparing these two techniques for investigating dimensional interaction, Wiener-Ehrlich (1978) concluded that the choice depends on the aim of the analysis. Dimensional analysis was considered to provide a more apt means of modeling human perception, whereas INDSCAL will reveal the structure of complex data. While Wiener-Ehrlich's synopsis is reasonable, it does not give due consideration to a very important aspect of human perception-that of intersubject variation. INDSCAL specifically permits each subject to weight the various dimensions differently before combining them, using a Euclidean metric to obtain an overall dissimilarity score. To achieve similar information using dimensional analysis would require individual subject-component interaction terms for the additivity model. INDSCAL clearly offers considerable advantage over dimensional analysis when the consensus of a group of subjects is required.

In this paper, we apply each of the three analyses to determine the analyzability of the two components of electrical stimuli; but, additionally, the variation in individual perception is examined closely. In the final section, the INDSCAL solution is used to derive a mathematical model of the configuration of stimuli given by the subjects.

The choice of electrocutaneous stimulation for the experimental study may seem unusual, as it has not been very widely used since the fundamental work relating physical scales of shock to subjective estimates of intensity (Ekman, Frankenhaeuser, Levander, \& Mellis, 1964; Sternbach \& Tursky, 1969; Stevens, Carton, \& Schickman, 1958; and reinvestigated by McCallum \& Goldberg, 1975). The process has, however, been used to investigate the mechanism of pain (Higgins, Tursky, \& Schwartz, 1971; Swartz, 1953). The main advantage of electric shocks, as opposed to the more usual visual or auditory stimuli, was that the subjects were quite unfamiliar with the stimuli and components were not easy to describe verbally. This is the type of situation we expect in our work on meat flavor, for which these procedures

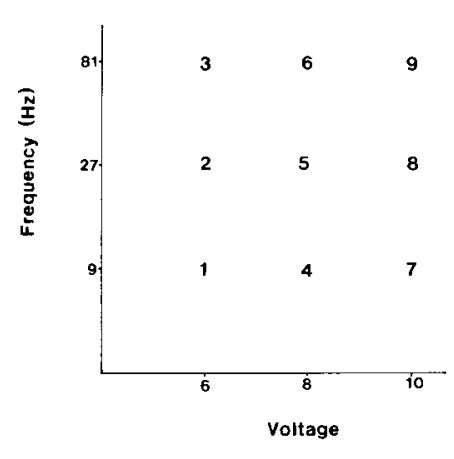

Figure 1. Plot of the nine electrocutaneous stimuli against frequency and voltage. Coordinates of frequency are plotted after transformation to the base 3 .

are ultimately to be used. A secondary advantage for pairwise difference tests was that the stimuli could be presented and evaluated very rapidly. The interval between successive stimuli in a pair was never more than $10 \mathrm{sec}$.

\section{METHOD}

\section{Stimulus Structure}

The variable components of the stimuli were voltage $(v)$ and frequency (f). Three levels each of voltage $\left(v_{1}, v_{2}, v_{3}=6,8\right.$, $10 \mathrm{~V}$, respectively) and frequency $\left(f_{1}, f_{2}, f_{3}=9,27,81 \mathrm{~Hz}\right.$ respectively) were selected. Preliminary experimentation (Thomson, 1981) had indicated that perceptual intervals between the three levels of voltage and between the three levels of frequency were of the same order. By combining all levels of both components, nine physically different stimuli were produced and numbered 1 to 9 as follows: $v_{1} f_{1}, v_{1} f_{2}, v_{1} f_{3}, v_{2} f_{1}, v_{2} f_{2}, v_{2} f_{3}, v_{3} f_{1}, v_{3} f_{2}, v_{3} f_{3}$ (Figure 1). The 36 possible pairwise combinations of these stimuli are arranged by voltage interval and frequency interval in Table 1 , following the notation of Krantz and Tversky (1975). Thus, $V_{0}$ $\left(F_{0}\right)$ indexes pairs with the same voltage (frequency) level. Similarly, $V_{1}\left(F_{1}\right), V_{2}\left(F_{2}\right), V_{12}\left(F_{12}\right)$ index $v_{1} v_{2}\left(f_{1} f_{2}\right), v_{2} v_{3}\left(f_{2} f_{3}\right)$, $v_{1} v_{3}\left(f_{1} f_{3}\right)$ comparisons, respectively.

Table 1

The 36 Stimulus Pairs Arranged in Frequency Interval by Voltage Interval Matrix

\begin{tabular}{ccccc}
$\begin{array}{c}\text { Voltage } \\
\text { Interval }\end{array}$ & $\mathbf{F}_{\mathbf{0}}$ & $\mathbf{F}_{\mathbf{1}}$ & $\mathbf{F}_{\mathbf{2}}$ & $\mathbf{F}_{\mathbf{1 2}}$ \\
\hline \multirow{2}{*}{$\mathrm{V}_{\mathrm{o}}$} & & 1,2 & 2,3 & 1,3 \\
& & 4,5 & 5,6 & 4,6 \\
& & 7,8 & 8,9 & 7,9 \\
$\mathrm{~V}_{1}$ & 1,4 & 1,5 & 3,5 & 1,6 \\
& 2,5 & 2,4 & 2,6 & 3,4 \\
& 3,6 & & & \\
$\mathrm{~V}_{2}$ & 4,7 & 4,8 & 5,9 & 4,9 \\
& 5,8 & 5,7 & 6,8 & 6,7 \\
$\mathrm{~V}_{12}$ & 6,9 & & & \\
& 1,7 & 1,8 & 2,9 & 1,9 \\
& 2,8 & 2,7 & 3,8 & 3,7 \\
\hline
\end{tabular}

Note-Stimuli are numbered as in Figure 1. Subscripts for intervals are defined as follows: $0=$ no difference; $I=$ low $/$ medium; 2 = medium $/$ high $; 12=$ low $/$ high . 


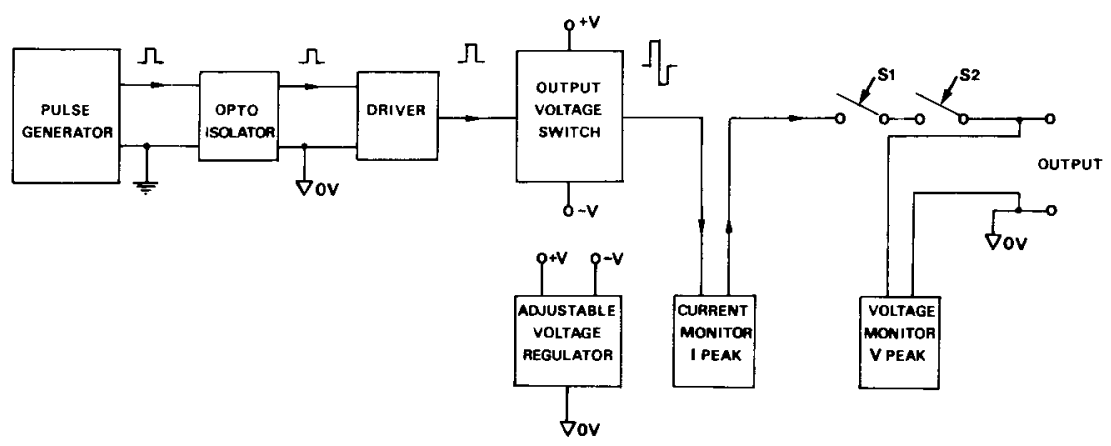

Figure 2. Apparatus used to generate electrocutaneous stimuli.

\begin{abstract}
Apparatus
Stimuli were generated using the unit shown diagrammatically in Figure 2. This unit produced a constant-voltage bidirectional square-wave output and met the following basic specification: output voltage, adjustable from 2 to $25 \mathrm{~V}$; frequency range, adjustable from 0 to $100 \mathrm{~Hz}$; output resistance, $2 \mathrm{k}$. The pulse generator produced a variable-pulse-repetition frequency of even mark to space ratio. Thus, the mean voltage per unit time was constant irrespective of frequency. The stimuli were applied across the first and second fingers of the subject's left hand by means of two separate electrodes, which simply comprised plastic tubes fitted with an electrical terminal and containing $.001 \mathrm{~N}$ salt solution $(\mathrm{NaCl})$. Since the electrical resistance of human skin is extremely variable, to introduce some measure of standardization among subjects and between sessions, the volume of electrolyte was adjusted after a 5 -min equilibration period to give a stimulus current of $.45 \mathrm{~mA}$ at $8 \mathrm{~V}$ and $9 \mathrm{~Hz}$.
\end{abstract}

\section{Subjects}

Nine subjects, five male and four female, were recruited from among the staff at the Meat Research Institute. All of the subjects had participated in preliminary experiments of this type, but it must be emphasized that no formal training or direction was given. The subjects were deliberately kept unaware of the precise nature of the stimuli and object of the experiment.

\section{Procedure}

The apparatus was arranged on a desk top and concealed from the subject by a screen. To administer the stimulus, both the experimenter and the subject were required to depress separate switches concurrently. This was considered an important psychological precaution when dealing with a noxious stimulus such as electric shock. In practice, the most intense stimulus $(10 \mathrm{~V}, 81 \mathrm{~Hz})$ was not judged to be unpleasant. Once the subjects were familiar with the range of stimuli, the stimulus was initiated by the subject and terminated by the experimenter after $3 \mathrm{sec}$. This ensured that the subjects received the same magnitude of stimulus.

The subjects were required to give a number reflecting the overall perceived difference within each pair of stimuli. No constraints on magnitude or direction were imposed. The interval between the two stimuli in each pair and between successive pairs was 10 and $30 \mathrm{sec}$, respectively. The order of stimulus presentation was initially random, but thereafter was maintained across all subjects and replicates. The subjects attended five sessions, of which the first two were regarded as preliminary to permit them to become consistent in their use of number for scoring differences. Only the last three sessions were analyzed and are reported in this paper.

\section{ANALYSES AND RESULTS}

\section{Dimensional Analysis}

For dimensional analysis, the grouping of stimulus pairs presented in Table 1 may be expressed as a complete within-subjects factorial design with the following main effects: voltage interval (V), frequency interval (F), and stimulus pairs (P), nested within each $(V \times F)$ combination $[P(V \times F)]$. Sessions (R) may be regarded as blocks.

Wiener-Ehrlich (1978) advocated that interdimensional additivity should be tested by examining the significance of stimulus pair differences $[P(V \times F)]$ for each set of stimulus pairs exhibiting unidimensional variation (in this experiment, two sets of nine pairs represented by the first row and column of Table 1), and for the set of stimulus pairs exhibiting bidimensional variation (in this case, the 18 remaining pairs). The intradimensional subtractivity property requires that the ordering of differences along one component dimension be independent of the interval in any other dimension. Again, adopting WienerEhrlich's interpretation, this will be manifested as a nonsignificant interaction term $(V \times F)$ in the bidimensional pair analysis of variance.

\section{Unidimensional Pairs}

Figure 3 shows the scores given by each subject (averaged across the three sessions) for the voltage interval differences at the three levels of frequency. Similarly, Figure 4 shows subjects' scores for frequency interval differences at the three levels of voltage. These figures clearly reveal the large variation in perceptual scoring patterns displayed by the subjects. For example, in Figure 3, scores at the median level of frequency are lower than the other two levels in at least one of the $V_{1}, V_{2}, V_{12}$ comparisons for seven of the nine subjects. The judgments made by Subjects 4, 6, and 8 seem to be particularly influenced by this level of frequency in the $V_{12}$ comparisons. In contrast, Subjects $1,2,3,7$, and 9 show an increasing augmentation effect in the perception of low-high voltage differences with increasing frequency. In Figure 4, the augmentation effect of voltage level on perceived frequency interval is apparent in all comparisons $\left(F_{1}, F_{2}, F_{12}\right)$.

To assess the extent of interdimensional augmentation or reduction effects, the procedure described by Krantz and Tversky (1975) was adopted. This pro- 


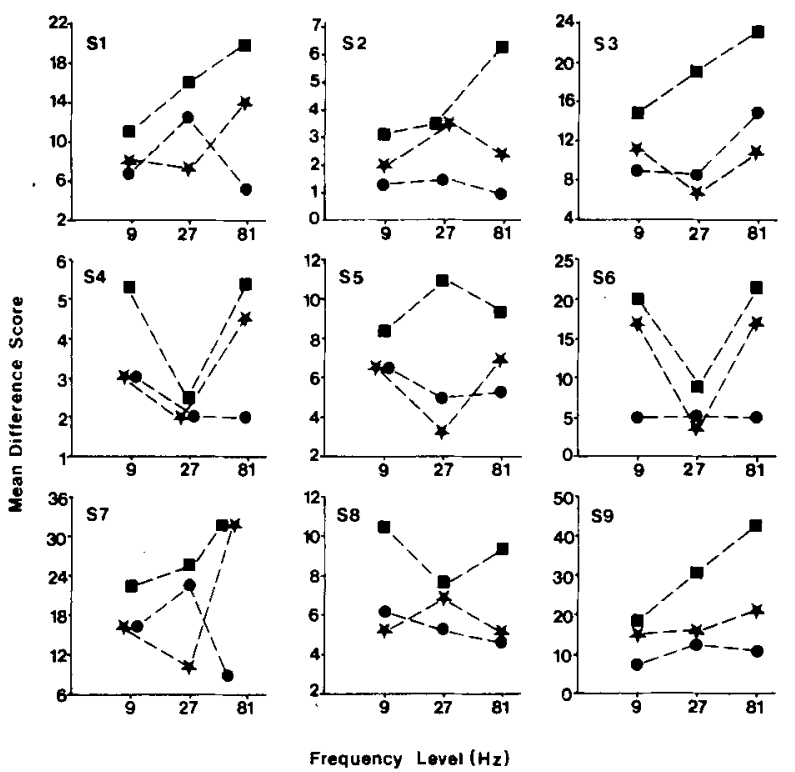

Figure 3. Unidimenslonal pairs. Mean difference scores for nine subjects for three voltage-frequency Intervals plotted agalnat voltage level. Key: $\bullet, 6$ vs. 8 V (V1); $\star, 8$ vs. 10 V (V2); $\square, 6$ vs. $10 \mathrm{~V}$ (V12).
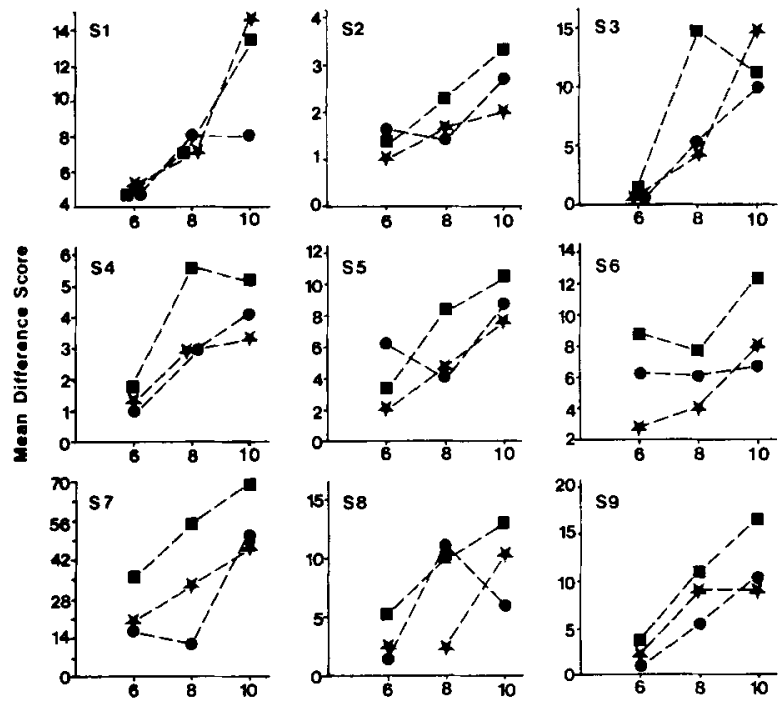

Voltage Level (Volts) quency interval scores contrasts with the rather ambivalent effect of frequency level on voltage interval scores.

Analysis of variance confirmed that property of interdimensional additivity was not upheld and showed a statistically significant $(p<.01)$ difference between stimulus pairs within $\mathrm{V}$ or $\mathrm{F}$ means square for almost all subjects. The frequency interval scores of Subject 6 was a notable exception.

Finally, the relative weight given by subjects to the $\mathrm{V}$ and $\mathrm{F}$ comparisons is estimated by comparing the magnitude of the distances given to the two interval scales (vertical axes in Figures 3 and 4). The majority of subjects estimated larger distances for $V_{12}$ comparisons than for the $F_{12}$ comparisons; the augmentation effect of frequency on voltage interval may be partly responsible in some subjects. Exceptions to this general pattern were Subjects 7 and 8, who tended to score frequency intervals higher than voltage intervals. These results will be considered further when a comparison is made between them and the relative weightings obtained in the INDSCAL solution.

\section{Bidimensional Pairs}

Having established that the distance scores for frequency and voltage intervals were not entirely independent, the bidimensional pairs were examined to determine the manner in which subjects synthesize their perception of interval differences along two dimensions into a single score. Again, graphical displays were used.

In Figures 5 and 6, the bidimensional cell means are illustrated as a function of difference on one dimension ( $V$ in Figure 5, $F$ in Figure 6) while the other dimension is held constant. Care is required in the interpretation of these graphs, so the various behavior patterns are discussed in detail.

Consider Subject 3. In Figure 6, this subject's scores increase sharply at all three frequency intervals as

Table 2

Equality Tests for $\mathbf{V} \times \mathbf{F}$ Dimensions

\begin{tabular}{cccccc}
\hline & \multicolumn{2}{c}{$\begin{array}{c}\text { Frequency } \\
\text { Comparisons }\end{array}$} & & \multicolumn{2}{c}{$\begin{array}{c}\text { Voltage } \\
\text { Comparisons }\end{array}$} \\
\cline { 2 - 3 } \cline { 5 - 6 } Subject & + & & & + & - \\
\hline 1 & 5 & 0 & 4 & 2 \\
2 & 5 & 1 & 3 & 2 \\
3 & 5 & 1 & 2 & 2 \\
4 & 5 & 1 & 2 & 3 \\
5 & 6 & 0 & 3 & 3 \\
6 & 5 & 1 & 4 & 1 \\
7 & 5 & 1 & 4 & 2 \\
8 & 4 & 1 & 3 & 1 \\
9 & 5 & 0 & 4 & 1 \\
\hline
\end{tabular}

Note-Entries are the number of times a subject gives a larger $(+)$ or smaller $(-)$ distance score for a particular comparison interval at the next highest absolute level of the other (constant) dimension.
Figure 4. Unidimendonal pairs. Mean difference scores for ulne subjects for three voltage-frequency intervals plotted againut voltage level. KEY: $\bullet$, 9 ve. 27 Hz (F1); $\star, 27$ vs. 81 Hz (F2); 0,9 vs. $81 \mathrm{~Hz}$ (F12).

cedure simply involves enumeration of the number of occasions in which a particular comparison $\left(\mathbf{F}_{1}\right.$, for example) increases or decreases at the next highest level of the other dimension (e.g., low to medium to high voltage). Table 2 shows the results of applying this test of equality to the data in Figures 3 and 4. The considerable influence of voltage level on fre- 

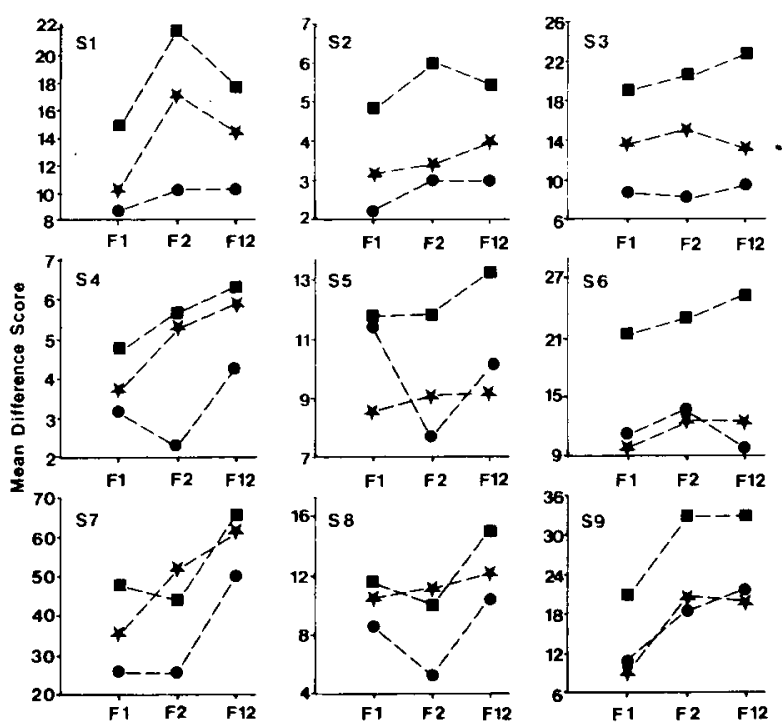

Frequency Interval

Figure 5. Bidimensional pairs. Mean difference scores for nine subjects for three voltage intervals, plotted against frequency interval. KEY: $\bullet, 6$ vs. 8 V (V1); $\star, 8$ vs. $10 \mathrm{~V}$ (V2); $\square, 6$ vs. $10 \mathrm{~V}(\mathrm{V12})$.
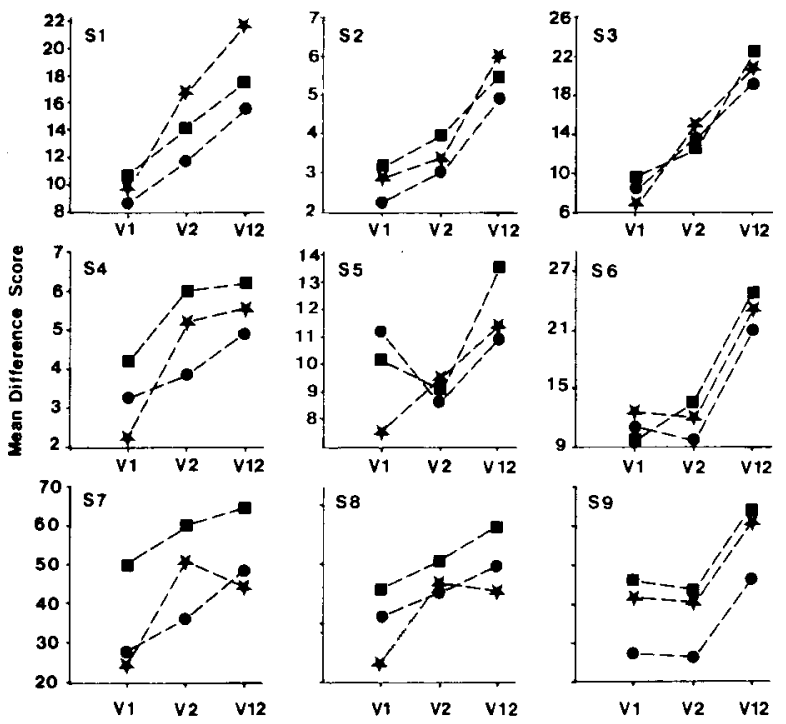

Voltage Interva

Figure 6. Bidimensional pairs. Mean difference scores for nine subjects for three frequency intervals plotted against voltage interval. KEY: $\bullet, 9$ vs. $27 \mathrm{~Hz}$ (F1); $\star, 27$ vs. $81 \mathrm{~Hz}$ (F2);, 9 vs. $81 \mathrm{~Hz}$ (F12).

voltage interval changes from $V_{1}$ to $V_{2}$ to $V_{12}$. The corresponding graph in Figure 5 shows an upward scoring trend with change in frequency interval $\left(F_{1}\right.$, $F_{2}, F_{12}$ ) only at the highest voltage interval. We conclude that this subject finds the voltage intervals monotonically increasing and is only marginally influenced by frequency difference. With respect to voltage interval, the behavior patterns of Subjects 1 and 2 are similar. In contrast, Subject 9 gave the same scores for $V_{1}$ and $V_{2}$ at constant frequency intervals (Figure 5), and it is concluded that she found $V_{1}$ and $V_{2}$ to be perceptually equal. The same is also true for $F_{2}$ and $F_{12}$ at constant voltage intervals (Figure 6). The scoring patterns of Subjects 7 and 8, who weighted frequency more heavily than voltage in the unidimensional pairs, are very similar. $F_{1}$ and $F_{12}$ scores are monotonically increasing with $V_{1}, V_{2}$, $V_{12}$, whereas a slight decrease in $F_{2}$ is observed between $V_{2}$ and $V_{12}$. Almost the exactly opposite pattern is revealed in Figure 6.

Figures 3-6 provide a useful means of illustrating differences in the scoring patterns of subjects, and from these it is possible to form subgroups of subjects who score similarly.

Analysis of variance of the bidimensional pairs confirmed the nonadditivity of dimensions, giving a significant "between pairs within voltage interval $x$ frequency interval mean square" for each subject. The test for significant deviance from intradimensional subtractivity [significance of $\mathrm{V} \times \mathrm{F}$ interaction term against $P(V \times F)$ ] was not upheld, and it is concluded that, at the ordinal level, intervals $V_{12}$ and $F_{12}$ were generally perceived as being larger than $\left(V_{1}, V_{2}\right)$ or $\left(\mathrm{F}_{1}, \mathrm{~F}_{2}\right)$.

To summarize this section, the hypothesis of interdimensional additivity was rejected, although perception of differences in one dimension was reasonably compatible at the ordinal level. Investigation of the bidimensional pairs indicated that the two components are combined by subjects in a complex manner. The disparity in scoring behavior appears to preclude the simple averaging of subject scores.

\section{Metric Structure}

The results of the previous section suggest that the two components of the stimuli lie somewhere between being completely analyzable and totally integral. The experience of Wiener-Ehrlich (1978) led us to expect that a Euclidean metric (which appears reasonably robust to all but the most severe violations of metric assumptions) will normally give lower stress values than city block will.

The multidimensional scaling program KYST-2 (Kruskal, 1976) was used to fit Euclidean and city block metrics to the distance matrices of each subject at each session. The stress values for twodimensional solutions are given in Table 3. To avoid the problem of local optima (particularly prevalent with city block metric), the stress values quoted are the minimum value from five runs, three using a randomly selected starting configuration and two using the TORSCA option-an adaption of the classical metric Torgerson technique used to derive a starting configuration. The TORSCA runs usually gave the minimum stress value for the city block 
Table 3

Stress Values (Formula 1) For Two-Dimensional KYST Solutions Using Euclidean (E) and City Block (CB) Metrics

\begin{tabular}{|c|c|c|c|c|c|c|}
\hline \multirow{2}{*}{$\begin{array}{l}\text { Sub- } \\
\text { ject }\end{array}$} & \multicolumn{2}{|c|}{ Session 1} & \multicolumn{2}{|c|}{ Session 2} & \multicolumn{2}{|c|}{ Session 3} \\
\hline & $\mathrm{E}$ & CB & $\mathrm{E}$ & CB & E & $\mathrm{CB}$ \\
\hline 1 & .108 & .101 & .104 & .102 & .096 & .084 \\
\hline 2 & .059 & .042 & .030 & .035 & .069 & .050 \\
\hline 3 & .084 & .080 & .034 & .058 & .037 & .034 \\
\hline 4 & .053 & .051 & .024 & .009 & .043 & .038 \\
\hline 5 & .092 & .080 & .116 & .089 & .123 & .096 \\
\hline 6 & .016 & .009 & .056 & .045 & .080 & .113 \\
\hline 7 & .076 & .087 & .107 & .088 & .069 & .066 \\
\hline 8 & .093 & .070 & .068 & .102 & .088 & .120 \\
\hline 9 & .046 & .029 & .061 & .027 & .044 & .009 \\
\hline
\end{tabular}

metric. Replicate runs using the Euclidean metric invariably gave very similar stress values.

The stress values in Table 3 indicate that there is little to choose between the two different metrics and supports Wiener-Ehrlich's (1978) conclusion that investigation of metric structure rarely gives a definite answer.

\section{Individual Differences Scaling}

INDSCAL (Carroll \& Chang, 1970) postulate an underlying configuration of stimuli which is "modified" by the individual subjects, in that they may weight the underlying dimensions differently in perceptual scaling. Assuming a Euclidean metric, INDSCAL derives the coordinates of the stimuli relative to a number of dimensions (specified by the experimenter) for the "group" space which has equal weights on each of the specified dimensions. At the same time, the subject weightings are estimated to enable each "private" space to be calculated. The normalized squared score $(i, j)$ given by the subject $k$ to the distance between stimuli $i$ and $j$ is modeled as:

$$
d(i, j,)=\sum_{m=1}^{a} w_{k m}\left(x_{i m}-x_{j m}\right)^{2},
$$

where $W_{k m}$ is the weight given by subject $k$ to the $\mathrm{m}^{\text {th }}$ dimension of the INDSCAL solution and $\mathrm{x}_{\mathrm{im}}$, $\mathbf{x}_{j m}$ are the coordinates of the stimuli relative to this dimension. In this form, it constitutes the normal three-way INDSCAL analysis. When replicate sessions are included for each subject, it is possible to extend to a four-way analysis, estimating an extra set of weights, one for each session on each dimension of the group space.

The version of INDSCAL used to analyze the electrocutaneous stimuli data was implemented as part of the MDS(X) multidimensional scaling suite (Coxon, Jones, Muxworthy, \& Prentice, Note 1). Figure 7 illustrates the configuration of stimuli in the group space obtained by a four-way INDSCAL run, on the complete data (nine subjects on each of three sessions). The scales of each dimension have been standardized, as was each individual input matrix. The "goodness of fit" of the solution may be judged from the correlations of the computed private configuration of each subject, with the given distance matrices. These appear in Table 4 and are satisfactory $(p<.001)$. Further confirmation appears in the two ancillary plots of session and subject weights (Figure 8), when the square of the distance of each point from the origin can be loosely interpreted as the percentage of variance in the data (to which that point refers) accounted for by the INDSCAL solution. The three points $A, B$, and $C$, which refer to the three sessions, are clustered at the top right corner, indicating good recovery by INDSCAL and equal weighting on the two dimensions of the group space in all three sessions. The distance of the subject points from the origin are all of the same order, suggesting that the two-dimensional plot (Figure 7) is representative of all subjects. The INDSCAL fit is particularly encouraging because it shows the considerable variation anticipated in subject weights. Three categories of behavior emerge: Subjects 1, 2, 3,6 , and 9 weight heavily on the horizontal dimen-

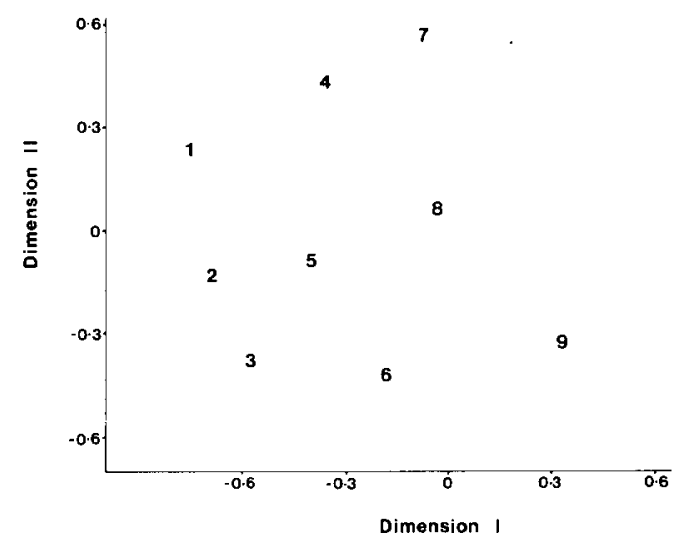

Figure 7. Two-dimensional configuration of electrocutaneous stimuli in the group space given by a four-way INDSCAL. Numbering of stimuli is as in Figure 1.

Table 4

Correlations Between INDSCAL Solution and Original Data for Each Session of Each Subject

\begin{tabular}{cccc}
\hline Subject & Session 1 & Session 2 & Session 3 \\
\hline 1 & .83 & .83 & .84 \\
2 & .81 & .94 & .85 \\
3 & .76 & .81 & .90 \\
4 & .82 & .87 & .85 \\
5 & .79 & .82 & .77 \\
6 & .87 & .91 & .83 \\
7 & .71 & .84 & .79 \\
8 & .81 & .87 & .88 \\
9 & .85 & .89 & .90 \\
\hline
\end{tabular}

Note-Correlations greater than .52 are statistically significant $(p<.001$ ) on 34 degrees of freedom ( 36 distances given at each session). 


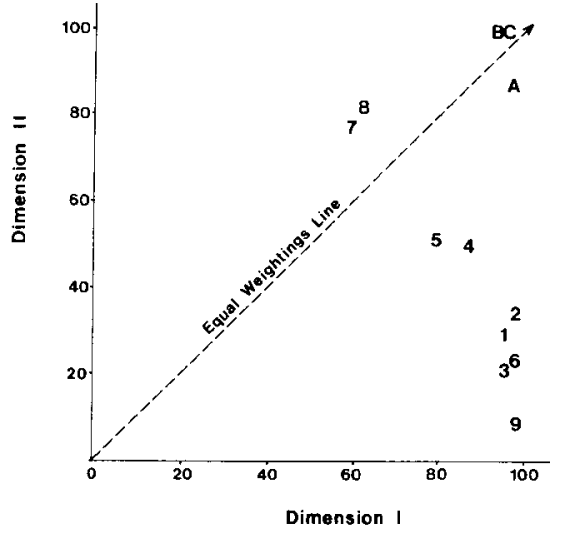

Figure 8. Two-dimensional configuration of Sessions 1, 2 and 3 (denoted by A, B, C, respectively) and subject (denoted by digits 1 to 9), defined by their relative weightings on the dimensions of the group space.

sion, Subjects 4 and 5 weight the horizontal dimension slightly more than the vertical, and Subjects 7 and 8 weight the vertical more than the horizontal. These weights correspond fairly well to the relative weightings deduced from Figures 3 and 4.

The INDSCAL solution supports the results of dimensional analysis; the interdimensional additivity hypothesis is clearly violated, whereas the property of intradimensional subtractivity cannot be rejected. The correlation between the dimensions is small $(-.06)$, and so the dimensions may be interpreted as voltage (horizontal) and frequency (vertical). The direction of these dimensions is arbitrary; it is the internal structure that is relevant.

\section{Deriving a Psychophysical Model}

As it seemed reasonable to interpret the two dimensions of the INDSCAL solution as direct psychophysical correspondents of voltage and frequency, the next logical step was to use these dimensions to investigate the synthesizing operations performed by subjects in their judgments of dissimilarity of the bidimensional pairs. The aim was to derive a mathematical model that would estimate the observed squared distance $\delta(i, j$,$) between bidimensional stim-$ uli based on the scores given to the corresponding unidimensional pairs. For example, the distance between stimuli 1 and 5 would be expressed as a function of the distance between stimuli 1 and 4 (voltage $v_{1}$ to $v_{2}$ at constant $f_{1}$ ) and stimuli 1 and 2 (frequency $f_{1}$ to $f_{2}$ at constant $v_{1}$ ). Algebraically, the observed squared distance between stimuli 1 and 5 may be written as

$$
\delta(1,5)=\delta\left(v_{1} f_{1}, v_{2} f_{2}\right)
$$

and estimated as

$$
D(1,5)=D\left(v_{1} f_{1}, v_{2} f_{2}\right) \text {. }
$$

The simple Euclidean combinatorial model assumes that the distance may be expressed according to the Pythagorean rule:

$$
\begin{aligned}
D\left(v_{1} f_{1}, v_{2} f_{2}\right) & =D\left(v_{1} f_{1}, v_{2} f_{1}\right)+D\left(v_{1} f_{1}, v_{1} f_{2}\right) \\
& =D(1,4)+D(1,2) .
\end{aligned}
$$

If the horizontal and vertical axes of the group space (Figure 7) are used to represent voltage and frequency scales, respectively, Equation 5 may be expressed in terms of the coordinates as

$$
D\left(v_{1} f_{1}, v_{2} f_{2}\right)=\left(x_{1 v}-x_{4 v}\right)^{2}+\left(x_{1 f}-x_{2 f}\right)^{2},
$$

where $\left(x_{1 v}, x_{1 f}\right)$ refer to the coordinates of stimulus 1 on the $v$ and $f$ axes. The use of this model to predict bidimensional $\delta(1,5), \delta(2,6), \delta(4,8), \delta(5,9)$ squared distances among the electrical stimuli in the group space is shown in Figure 9. The dotted lines show the unidimensional distances used to calculate the corresponding bidimensional distance (continuous lines). The predicted position of the stimuli is shown by the bracketed numerals. The model clearly fits the data for the low-level comparison $[\delta(1,5) \bumpeq$ $D(1,5)]$. However, at higher voltage levels, the contribution of frequency difference $\left(x_{i f}-x_{j f}\right)$ is overestimated, and we may interpret this as representing the masking effect of voltage level on perception of frequency difference. In contrast, the voltage differ-

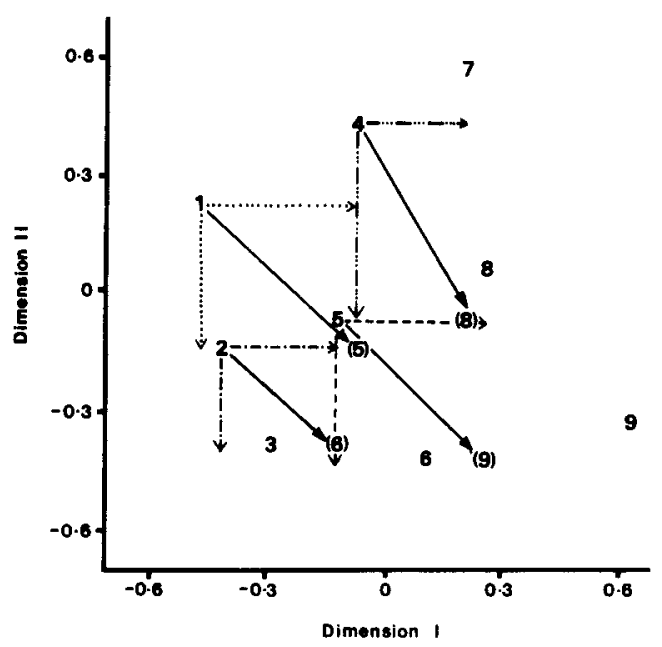

Figure 9. Prediction of bidimensional distences (continuous lines) in the group space using the Pythagoras rule to combine the corresponding unidimensional distances (broken lines). Predicted positions of the stimuli are shown as bracketed digits. 
ences are underestimated by the model at higher frequency level, representing the augmenting effect of frequency level on perception of voltage interval.

These observations suggest the following empirical equation to estimate the bidimensional distances.

$$
\begin{aligned}
D(1,5) & =D\left(v_{1} f_{1}, v_{2} f_{2}\right) \\
& =D^{(1)}\left(f_{1}, f_{2}\right)+D^{(1)}\left(v_{1}, v_{2}\right) \\
D(4,8) & =D\left(v_{2} f_{1}, v_{3} f_{2}\right) \\
& =(1-0.52) D^{(2)}\left(f_{1}, f_{2}\right)+D^{(1)}\left(v_{2}, v_{3}\right) \\
D(2,6) & =D\left(v_{1} f_{2}, v_{2} f_{3}\right) \\
& =D^{(1)}\left(f_{2}, f_{3}\right)+(1+.7) D^{(2)}\left(v_{1}, v_{2}\right) \\
D(5,9) & =D\left(v_{2} f_{2}, v_{3} f_{3}\right) \\
& =(1-.41) D^{(2)}\left(f_{2}, f_{3}\right)+(1+1.1) D^{(3)}\left(v_{2}, v_{3}\right)
\end{aligned}
$$

Here the superscript has been used to simplify notation for unidimensional comparisons, Thus, $D^{(1)}$ $\left(v_{1}, v_{2}\right)=\left(x_{2 v}-x_{1 v}\right)^{2}$ at level $f_{1}$. Equations 7 through 10 have been written in this form to correspond to Equation 6 of Krantz and Tversky (1975), who suggest the following combinatorial model for the squared distance $\delta\left(v_{i} f_{j}, v_{k} f_{m}\right)$.

$$
\begin{aligned}
\delta\left(v_{i} f_{j}, v_{k} f_{m}\right)= & F D\left(v_{i}, v_{k}\right)\left[1+\beta M\left(f_{j}, f_{m}\right)\right] \\
& +D\left(f_{j}, f_{m}\right)\left[1+\alpha M\left(v_{i}, v_{k}\right)\right] .
\end{aligned}
$$

$F$ is a function, strictly increasing in each of $v$ and $f$ taken separately. $D$ is the squared distance between two levels of a dimension at a constant value of the other dimension. $M$ is a location parameter expressed as a function of the subjective scales used to define $D$. The parameters, $\alpha, \beta$ are used to define the direction and magnitude of the interaction between dimensions. Positive values of $\alpha$ and $\beta$ produce augmentation, negative values produce reduction of distances with increasing values of the other dimension. Krantz and Tversky (1975) suggest the following form for $M$ (translated into our notation):

$$
M\left(v_{i} v_{j}\right)=\left(x_{i v}+x_{j v}\right) / 2 .
$$

The following equations can thus be derived to provide a solution set for $\alpha$ and $\beta$. From Equations 7 and 8 ,

$$
\alpha\left(\mathrm{x}_{1 \mathrm{v}}+\mathrm{x}_{2 \mathrm{v}}\right)=\beta\left(\mathrm{x}_{1 \mathrm{f}}+\mathrm{x}_{2 \mathrm{f}}\right)=0 .
$$

From Equation 8,

$$
\alpha\left(x_{2 v}+x_{3 v}\right)=-.62 .
$$

From Equation 9,

$$
\beta\left(x_{2 f}+x_{3 f}\right)=.6
$$

From Equation 10,

$$
\begin{gathered}
\alpha\left(x_{2 v}+x_{3 v}\right)=-.46 \\
\beta\left(x_{2 f}+x_{3 f}\right)=.90
\end{gathered}
$$

Examination of Equations 13 to 17 reveals that this model is inadequate. It is simple to satisfy Equation 13 for all $\alpha, \beta$ by rescaling the coordinates such that $x_{1 v}+x_{2 v}=x_{1 f}+x_{2 f}=0$. However, Equations $14,15,16$, and 17 are inconsistent. It is clear that the augmentation effect of frequency level on voltage difference is increasing, whereas the reduction effect of voltage level on frequency difference is decreasing. This suggests that $\alpha$ and $\beta$ are functions of voltage and frequency levels, respectively. We, therefore, propose an alternative model to predict distances of bidimensional comparisons:

$$
\begin{aligned}
\delta\left(v_{i} f_{j}, v_{k} f_{m}\right)= & \beta\left(f_{j}\right) D^{(j)}\left(v_{i}, v_{k}\right) \\
& +\alpha\left(v_{j}\right) D^{(i)}\left(f_{j}, f_{m}\right) .
\end{aligned}
$$

For the purpose of modeling the electrical stimuli, this model is constrained to distances between adjacent levels. The superscripts of $D$ are selected on the basis that $v_{i}<v_{k}, f_{j}<f_{m}$. Parameter estimates that will fit the INDSCAL bidimensional distances satisfactorily are:

$$
\begin{array}{lll}
\alpha\left(v_{1}\right)=1 & \alpha\left(v_{2}\right)=0.48 & \alpha\left(v_{3}\right)=0.59 \\
\beta\left(f_{1}\right)=1 & \beta\left(f_{2}\right)=1.70 & \beta\left(f_{3}\right)=2.10
\end{array}
$$

It is not possible to predict accurately the relationship between $\alpha$ and $v$ and between $\beta$ and $f$ with only three levels of each dimension.

\section{Interpreting the Model}

The results from INDSCAL and the modeling exercise of the previous section considerably enhance the conclusions from the dimensional analysis. Previously, it was only possible to state that the two components were not independent, although the rank orderings of levels within a component were maintained. The parameter estimates of $\alpha$ and $\beta$ for Equation 18 succinctly express the fact that, although differences in both components are perceptible at the levels used, the magnitude of difference perceived does depend on the level of the other component. The trend of the $\alpha$ and $\beta$ parameters with increasing intensity implies that at more extreme voltage levels this component will become dominant whatever the frequency level. 
The differences in the relative weightings on the components by the individual subjects may be due to a number of factors: personal biases, ad hoc subject hypotheses, and, in this experiment, disciplinary backgrounds may all contribute. However, it is not our aim to interpret this variation. The important point is that INDSCAL seeks to describe and incorporate this variation, and this is carried through into the model (Equation 18). This model will therefore be particularly useful in the investigation of stimuli that have strong hedonistic overtones and elicit considerable variation in response.

\section{DISCUSSION}

The purpose of this work was to investigate and model the observed interaction between voltage and frequency as concurrently varying components in a series of two-dimensional electrocutaneous stimuli. Dimensional analysis and analysis of metric structure both revealed a similar pattern of complex and mutual interaction between the two sensory components. Individual differences scaling (INDSCAL) revealed additional important differences in the nature and extent of the interaction apparent across subjects, enabling a generalized model representing the observed interaction to be derived.

Tversky and Krantz (1970) have suggested that there are two sets of scales that apply to each dimension perceived in the assessment of multidimensional stimuli. The first set of scales, termed psychophysical functions, applies directly to the physical input and defines the psychological scale along each dimension. The second set of scales, termed similarity functions, applies to the differences along each dimension as they are perceived in the assessment of overall dissimilarity between the multidimensional stimuli.

In terms of the INDSCAL model, the psychophysical scales are the coordinates $\mathbf{x}_{\mathrm{iv}}$ for voltage and $\mathbf{x}_{\mathbf{j f}}$ for frequency. The apparent interdimensional augmentation effect constrains us to consider these coordinates in triplets, within a fixed level of the other dimension. For example, $\left(x_{1 v}, x_{4 v}, x_{7 v}\right)$ form the triplet for voltage at the lowest level of frequency. The similarity functions are defined by the introduction of parameters $\alpha$ and $\beta$ and the formulation of Equation 18, which relates the two sets of scales.

One very appealing aspect of Equation 18 is that it applies equally well to the private space of any subject, because the estimates of $\alpha$ and $\beta$ are obtained from the squares of the ratios of distances along each dimension and will not be affected by rescaling into a "private space." The theoretical attractions of a model of this form are obvious. Estes (1975) writes: "What we hope primarily from models is that they will bring out relationships between experiments or sets of data that we would not otherwise have per- ceived. The fruit of an interaction between a model and data should be a new categorization of phenomena in which observations are organized in terms of a rational scheme in contrast to the surface demarcations manifest in data that have only come through routine statistical processing."

The derivation of a combinatorial model has profoundly increased our understanding of the underlying structure of these data and, hence, of the mode of interaction between voltage and frequency as stimulus components. The apparent augmentation effect in both psychophysical scales contrasts with the masking effect of voltage on the similarity scales of frequency.

The precision of the fitted parameters (Equation 18) for interpretation of the evaluative process in the perception of dissimilarities among two component electrocutaneous stimuli is limited by the few stimuli used. Getty, Swets, Swets, and Green (1979) validated an INDSCAL model by using it to predict confusion matrices of subjects in an independent identification task, but before such validation further investigations using more intervals that cover the complete perceptual range of both stimulus components are required.

\section{REFERENCE NOTE}

1. Coxon, A. P. M., Jones, C. L., Muxworthy, D. T., \& Prentice, M. J. The $M D S(X)$ series of multidimensional scaling programs (Report No. 37, Inter-University/Research Councils Series). Edinburgh: Program Library Unit, 1977.

\section{REFERENCES}

Canroll, J. D., \& Chano, J. J. Analysis of individual differences in multidimensional scaling via a $\mathrm{N}$-way generalization of "Eckart-Young" decomposition. Psychometrika, 1970, 35, 288-319.

Cohen, H. S., \& Jones, L. E. The effects of random error and subsampling of dimensions on recovery of configurations by non-metric multidimensional scaling. Psychometrika, 1974, 39, 69-90.

Ekman, G., Frankenhaeuser, M., Levander, S., \& Mellis, I. Scales of unpleasantness of electrical stimulation. Scandinavian Journal of Psychology, 1964, 5, 257-261.

Estes, W. K. Some targets for mathematical psychology. Journal of Mathematical Psychology, 1975, 12, 263-282.

GetTy, D. J., Swet8, J. A., Swet8, J. B., \& Green, D. M. On the prediction of confusion matrices from similarity judgments. Perception \& Psychophysics, 1979, 26, 1-19.

Higains, J. D., Tursky, B., \& SChwartz, G. E. Shock elicited pain and its reduction by concurrent tactile stimulation. Science, $1971,172,866-867$.

Krantz, D. H., \& Tversky, A. Similarity of rectangles: An analysis of subjective dimensions. Journal of Mathematical Psychology, 1975, 12, 4-34.

Kruskat, J. B. Multidimensional scaling and other methods for discovering structure. In K. Enslein, A. Ralston, \& H. Wilf (Eds.), Statistical methods for digital computers. New York: Wiley, 1976.

MCCallum, P., \& Goldbero, H. Magnitude scales for electrocutaneous stimulation. Perception \& Psychophysics, 1975, 17, 75-78. 
Shepard, R. N. Attention and the metric structure of the stimulus space. Journal of Mathematical Psychology, 1964, 1, 54-87. Sternbach, R. A., \& Tursky, B. On the psychophysical power function in electric shock. Psychonomic Science, 1969, 1, 217-218. Stevens, S. S., Canton, A. S., \& Schickman, G. M. A scale of apparent intensity of electric shock. Journal of Experimental Psychology, 1958, 56, 328-334.

Swartz, P. A new method of scaling pain. Journal of Experimental Psychology, 1953, 45, 288-293.

Thomson, D. M. H. An investigation of non-verbal sensory techniques for evaluating meat flavour. Doctoral thesis, University of Bristol, 1981.
Tver8ky, A., \& Krantz, D. H. Similarity of schematic faces: A test of interdimensional additivity. Perception \& Psychophysics, $1969,5,124-128$.

Tversky, A., \& Krantz, D. H. The dimensional representation and the metric structure of similarity data. Journal of Mathematical Psychology, 1970, 7, 572-596.

WiEner-EhrLich, W. K. Dimensional and metric structures in multidimensional stimuli. Perception \& Psychophysics, 1978, 24, 399-414.

(Manuscript received March 24, 1981; revision accepted for publication August 19, 1981.) 